\title{
FORMALIZING THE OPTIMIZATION PROBLEM IN LONG-TERM CAPABILITY PLANNING
}

\author{
Todor TAGAREV, Tsvetomir TSACHEV, and Nikolay ZHIVKOV
}

\begin{abstract}
In defining future force capabilities, decision makers need to balance objectives, strategy, force capabilities, and risk within a forecasted force development environment and resource levels. This is commonly a task in long-term defense and force planning. This paper presents a mathematical formalization of the planning problem that involves static and dynamic optimization. Several static and dynamic discrete optimization models illustrate the approach. The concluding section presents a short deliberation on applicability.
\end{abstract}

Keywords: Capability-Based Planning, CBP, scenario planning, force structure, defense planning risks, discrete dynamic optimization.

\section{Summary on Capability-Based Planning}

The armed forces of a country are designed so that they would be able to meet future challenges and threats. Since the end of the Cold War there is no single threat that drives the force planning process. On the contrary, the armed forces are expected to perform a very broad spectrum of missions in diverse situations, or scenarios. Therefore, prevailing approaches to defense and force planning at present do not attempt to optimize the future force structure against one or a few threats; instead the purpose is to define a force structure that would provide for robust performance of various missions in uncertain conditions. ${ }^{1}$

In the definition of future force capabilities defense policy makers and planners seek a balance among four main variables: policy objectives, strategy, means, and planning risk, accounting for trends in the security environment and anticipated availability of resources. This balance is sought in a cyclic process (see Figure 1), known also as the "Bartlett model."2

In the examination of "means" our focus in this case is on force capabilities. For a broader and more comprehensive understanding of "means" necessary to meet defense policy objectives refer to a most recent work of one of the authors. ${ }^{3}$ 


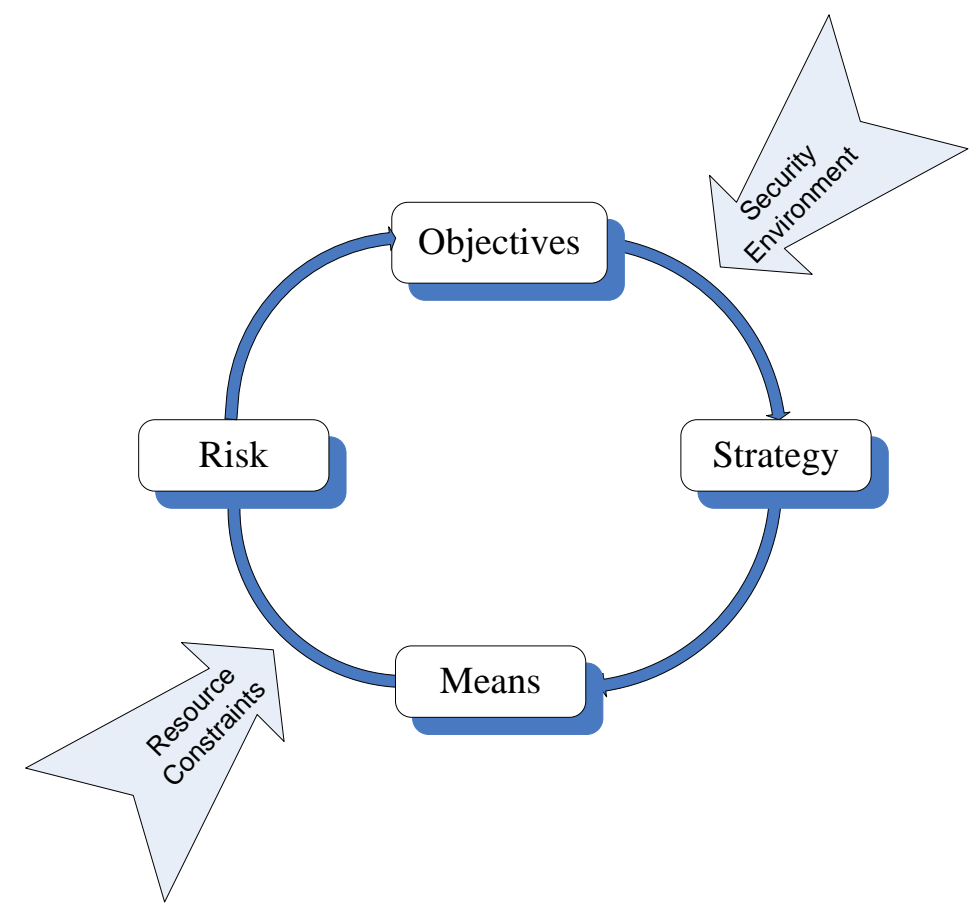

Figure 1: Balancing Security Objectives, Strategy, Means, and Planning Risks.

A capability is defined in relation to a task, performed under particular conditions, described in a planning scenario. ${ }^{4}$ The process of setting capability requirements is presented in Figure 2. A more detailed examination of the process shows the value of using standardized task lists (or "catalogue of tasks") in decomposing missions to tasks accounting for anticipated concepts of operation, as well as standardized capability partition in defining capability requirements (Figure 3). For the purposes of this study we assume that an adequate capability partition exists. ${ }^{5}$

Then, for each planning scenario force planners design force proposals in alternatives (Figure 4). To facilitate design efficiency and provide for compatibility with other planning processes, in advanced defense planning systems an effort is made to create and maintain a library of generic units (or modules). ${ }^{6}$

Cost-benefit analysis provides a possibility for rational selection of a particular force proposal, or subset of the force proposals, for each scenario (Figure 4). Minimization of planning risks ${ }^{7}$ under constraints is the key consideration in aggregating force proposals across planning scenarios. ${ }^{8}$ 


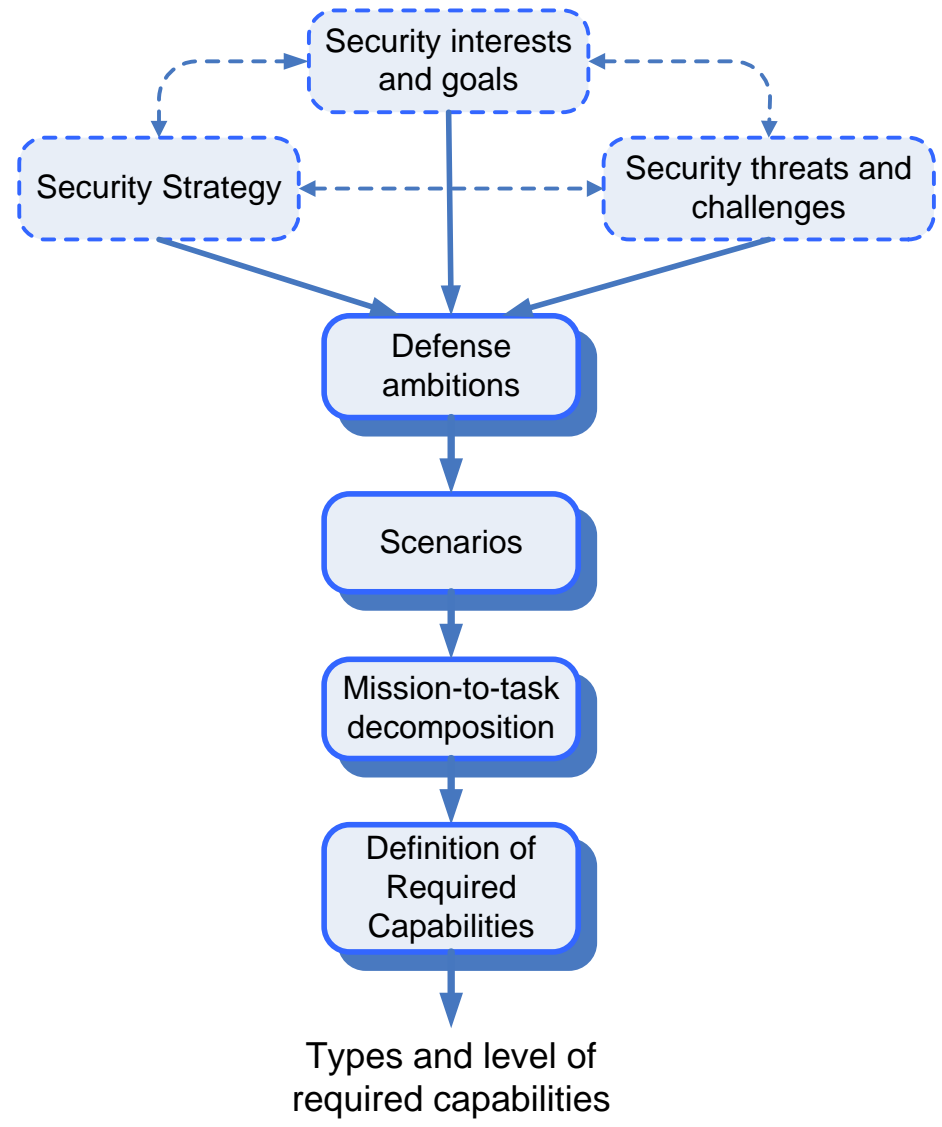

Figure 2: Main Steps in Defining Capability Requirements.

Thus, the long-term force planning problem may be construed as finding a future force structure that carries the capabilities minimizing planning risks over a set of planning scenarios. The issue of generation of defense planning scenarios, the selection of the set of scenarios to be included in the planning process, as well as the hypotheses for simultaneous or near simultaneous realization of two or more scenarios is not examined here. ${ }^{9}$ The following sections provide detail on possible definitions of the respective optimization problem.

\section{Formulation of an Optimization Problem}

Given is a number of planning scenarios. Each scenario describes the conditions for realization of specific threats and/or challenges to the armed forces and is used to define their structure and readiness levels. For the purposes of this study we assume that 


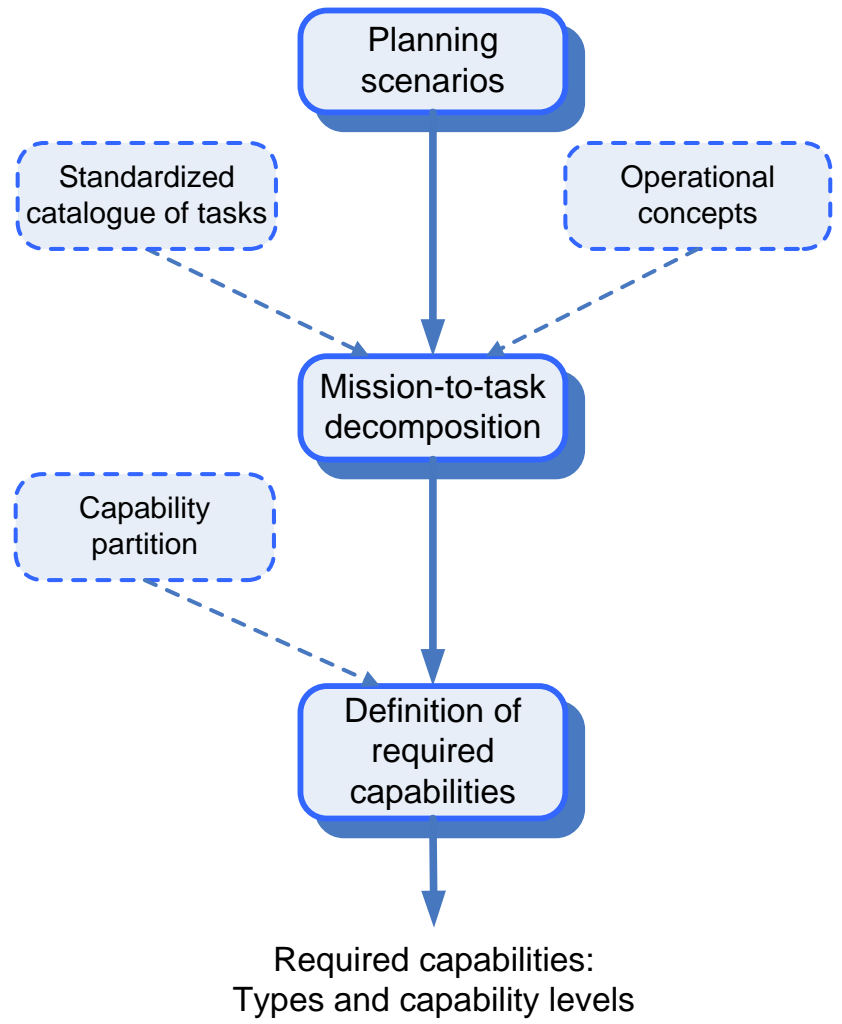

Figure 3: Linking Scenarios to Tasks to Required Capabilities.

the probability of occurrence of each scenario is known, as well as the challenges it poses in terms of capabilities required for adequate performance in case the scenario realizes. An abstract weight is used to measure the importance of each scenario. It is defined by experts.

The armed forces consist of units of different types. For each unit type the predefined measurable (quantifiable levels of) capabilities are known. Various force structures can be formed by units of different types. The numbers of available units of each type in the force structure are subject to optimization. The capability measures of armed forces as a whole are also given by a vector of capabilities whose components usually are not sums of the capabilities of all units included. The negative consequences resulting from the occurrence of each scenario are defined as functions dependent on the proposed force structure. 


\section{Planning scenario $\mathrm{m}$}

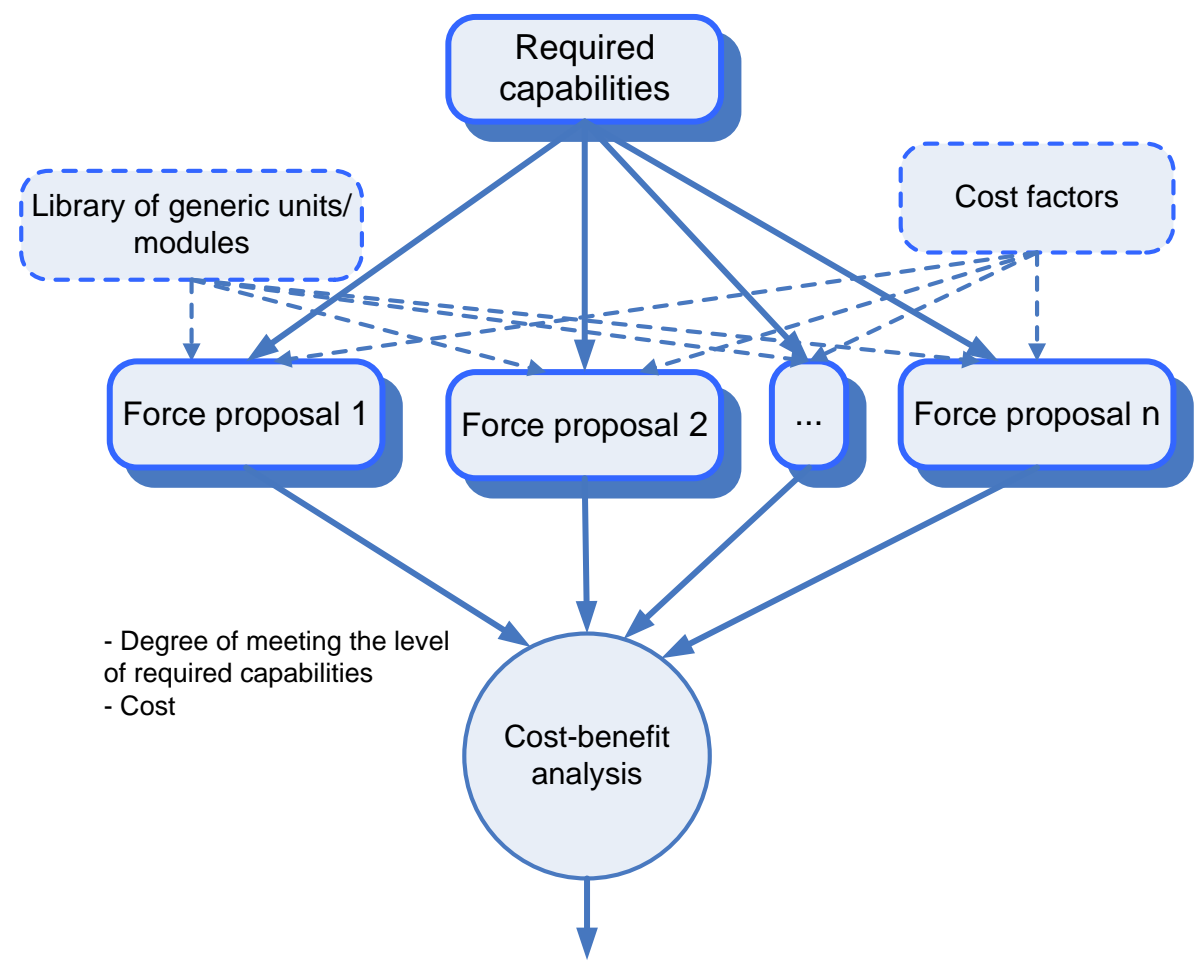

Cost-effective alternatives

Figure 4: Designing and Assessing Force Alternatives.

The models present an approach through discrete optimization problems to find structures of armed forces and are aimed to optimize these structures so that at minimal cost the threats (the challenges) are neutralized. Another circle of problems, that we consider, concerns the minimization of the negative consequences from the occurrences of the scenarios under budget restrictions, i.e. the money spent to obtain, maintain, upgrade, or disband units of different types.

The models can be used to formally assess the long-term planning not only of the whole structure of the armed forces but also for any of its substructures. Herein we do not aim at tackling in detail the issues related to numerical solutions of the respective optimization problems. 


\section{Mathematical Formalization Scheme}

Consider

$l$ scenarios $S_{1}, S_{2}, \ldots, S_{l}$;

(It is assumed some of the scenarios in the list to be occurrences of two different scenarios, i.e. scenario $S_{3}$, for instance, might be " $S_{1}$ and $S_{2}$ happen simultaneously")

$n$ - capabilities required from the armed forces: $C_{1}, C_{2}, \ldots, C_{n}$, with required levels of these capabilities $\overline{c_{1}}, \overline{c_{2}}, \ldots, \overline{c_{n}}$

(The required capabilities and their required (measurable) levels are determined here by all scenarios);

$q$ - types of units $U_{1}, U_{2}, \ldots, U_{n}$

and

force-structures $\left(u_{1}, u_{2}, \ldots, u_{q}\right)$ where $u_{i}$ is the number of units of type $U_{i}$ in the structure for $i=1,2, \ldots, q$.

Each force-structure $\left(u_{1}, u_{2}, \ldots, u_{q}\right)$ generates a package (vector) of capabilities $\left(c_{1}, c_{2}, \ldots, c_{n}\right)$ where $c_{i}, 0 \leq c_{i}, i=1,2, \ldots, n$, is the level of capability $C_{i}$.

It is assumed that the measures (levels) of the capabilities are computable functions depending on the set of the forces structures:

$$
c_{i}=F_{i}\left(u_{1}, u_{2}, \ldots, u_{q}\right), \quad i=1,2, \ldots, n .
$$

The functions $F_{i}$ are not linear, but they are monotone with respect to any of their variables.

It is supposed also that for each scenario $S_{i}$ we are able to estimate the level of the negative consequences $N_{j}$, if $S_{j}$ occurs, as functions of the vectors of capabilities, i.e. given are

$$
N_{j}\left(c_{1}, c_{2}, \ldots, c_{n}\right), \quad j=1,2, \ldots, l \text {. }
$$

By virtue of (1), the functions $N_{j}$ can be viewed also as functions depending on the force structures, i.e.

$$
N_{j}\left(F_{1}\left(u_{1}, u_{2}, \ldots, u_{q}\right), \ldots, F_{n}\left(u_{1}, u_{2}, \ldots, u_{q}\right)\right), \quad j=1,2, \ldots, l .
$$


Denote the cost to acquire (and maintain for an abstract period of time $L$ ) a unit of type $U_{i}$ by $p_{i}, i=1,2, \ldots, q$.

Additional assumptions and notation will be made further down when necessary.

There are different ways to derive the values of the required capabilities $\overline{c_{i}}$ from all scenarios $S_{j}$. We consider briefly two of them.

Let $c_{i j}, i=1,2, \ldots, n, j=1,2, \ldots, l$, be the required level of capability $C_{i}$ for the scenario $S_{j}$. The numbers $\overline{c_{i}}$ are defined as follows:

i. $\overline{c_{i}}=\max \left\{c_{i j}: j=1,2, \ldots, l\right\}, \quad i=1,2, \ldots, n$.

ii. $\quad \overline{c_{i}}=\kappa_{i} \sum_{j=1}^{l} w_{j} c_{i j}$ for $i=1,2, \ldots, n$, where $w_{j}$ is the weight of scenario $S_{j}$ and all weights are normalized so that $\sum_{j=1}^{l} w_{j}=1$.

Here $w_{j}$ can be defined as a monotone function of the product $P_{j} I_{j} N_{j}$, $j=1,2, \ldots, l$, where $P_{j}$ is the probability of occurrence of the scenario $S_{j}, I_{j}$ is the expert given priority of $S_{j}$ and $N_{j}$ is the level of the negative consequences if the challenges of $S_{j}$ are not met.

The numbers $\kappa_{i}, \kappa_{i} \geq 1$, for $i=1,2, \ldots, n$, are roughly speaking "close to 1 " and they express the ambition, or a politically motivated will to have more of capability $C_{i}$ than necessary according to the integral criterion. They can be put also when the obtained by (ii) required capabilities are not sufficient for some specific scenarios of high priority or simply to better complement substructures of armed forces.

\section{Static Models}

In this section simplified models illustrating our approach are considered.

The following is a model of a force structure with capabilities $c_{i}$ not below given required levels of capabilities $\overline{c_{i}}, i=1,2, \ldots, n$, determined by all scenarios. The cost of the force structure is subjected to minimization. 


\section{Model 1}

$$
\sum_{i=1}^{q} p_{i} u_{i} \rightarrow \text { minimize }
$$

subject to the constraints

$$
\begin{aligned}
& F_{i}\left(u_{1}, u_{2}, \ldots, u_{q}\right) \geq \overline{c_{i}}, \quad i=1,2, \ldots, n, \\
& 0 \leq u_{i}, i=1,2, \ldots, q, \\
& u_{i}-\text { integer } i=1,2, \ldots, q .
\end{aligned}
$$

Let $\alpha_{j}, j=1,2, \ldots, l$, be acceptable levels of negative consequences resulting from occurrences of the scenarios $S_{j}, j=1,2, \ldots, l$. The next model minimizes the cost of force structure so that the negative consequences from any specific scenario $S_{j}$ do not exceed the acceptable level of negative consequences $\alpha_{j}$.

\section{Model 2}

$$
\sum_{i=1}^{q} p_{i} u_{i} \rightarrow \text { minimize }
$$

subject to the constraints

$$
\begin{aligned}
& N_{j}\left(F_{1}\left(u_{1}, u_{2}, \ldots, u_{q}\right), \ldots, F_{n}\left(u_{1}, u_{2}, \ldots, u_{q}\right)\right) \leq \alpha_{j}, \quad j=1,2, \ldots, l, \\
& 0 \leq u_{i}, i=1,2, \ldots, q, \\
& u_{i}-\text { integer } i=1,2, \ldots, q .
\end{aligned}
$$

Suppose weights $w_{j}$ are attributed to the scenarios $S_{j}$, measuring their importance for $j=1,2, \ldots, l$. The weights are derived, as discussed above, from the likelihoods (the probabilities) that the respective scenario will happen, and/or could be an expression of politically motivated prioritization of the scenarios, and/or could be influenced by the levels of the negative consequences if the threats or the challenges resulting from the specific scenario are not met.

Let $\alpha$ be an acceptable level of integrated negative consequences from all scenarios, for instance 


$$
\alpha=\sum_{j=1}^{l} w_{j} \alpha_{j}
$$

The model below is a variation of the previous model with one integral constraint.

\section{Model 3}

$$
\sum_{i=1}^{q} p_{i} u_{i} \rightarrow \text { minimize }
$$

subject to the constraints

$$
\begin{aligned}
& \sum_{j=1}^{l} w_{j} N_{j}\left(F_{1}\left(u_{1}, u_{2}, \ldots, u_{q}\right), \ldots, F_{n}\left(u_{1}, u_{2}, \ldots, u_{q}\right)\right) \leq \alpha_{j}, \quad j=1,2, \ldots, l, \\
& 0 \leq u_{i}, i=1,2, \ldots, q \\
& u_{i}-\text { integer }, i=1,2, \ldots, q .
\end{aligned}
$$

Model 3 has an advantage over Model 2 as the $l$ constraints in (2) are replaced by one constraint, cf. (3), and the drawback that the decision maker has to put weights on the scenarios.

Model 3 leads us to the following kind of a dual optimization problem where the integral estimate of the negative consequences is minimized and the cost of the force structure is bounded by (budget) restrictions.

\section{Model 4}

$$
\sum_{j=1}^{l} w_{j} N_{j}\left(F_{1}\left(u_{1}, u_{2}, \ldots, u_{q}\right), \ldots, F_{n}\left(u_{1}, u_{2}, \ldots, u_{q}\right)\right) \rightarrow \text { minimize }
$$

subject to the constraints

$$
\begin{aligned}
& \sum_{i=1}^{q} p_{i} u_{i} \leq b(b-\text { anticipated budget }), \\
& 0 \leq u_{i}, i=1,2, \ldots, q \\
& u_{i}-\text { integer }, i=1,2, \ldots, q .
\end{aligned}
$$




\section{Discrete Dynamic Models}

In real situations force planning is a process in which decisions are taken at different times (often by different people) and the planning is made in steps with specific goals and restrictions for each step while still there is one main goal to be achieved at the end of the planning period. Formalization of this process is described below.

Suppose a planning horizon of $T$ intervals of time each of length $L$ is given. For the sake of simplicity of the models below, we assume that the changes of the force structure occur at the beginning of every step, i.e. at the beginning of the time period $[(t-1) L, t L]$ for $t=1,2, \ldots, T$ where $T$ is a positive integer. The main objective is to have a force structure with a vector of capabilities dominating $\left(\overline{c_{1}}, \overline{c_{2}}, \ldots, \overline{c_{n}}\right)$ at $t=T$.

Let $\left(u_{1,0}, u_{2,0}, \ldots, u_{q, 0}\right)$ be the initial force structure before the process of long-term planning has started.

Suppose, for $i=1,2, \ldots, q$ and $t=1,2, \ldots, T$, that

$U_{i, t}$ is the number of available units of type $U_{i}$ at step $t$;

$p_{i, t}$ is the cost of maintaining a unit of type $U_{i}$ at step $t$;

$\bar{u}_{i, t}$ is the number of acquired units of type $U_{i}$ at step $t$;

$\bar{p}_{i, t}$ is the cost of acquiring a unit of type $U_{i}$ at step $t$;

$\tilde{u}_{i, t}$ is the number of disbanded units of type $U_{i}$ at step $t$;

$\tilde{p}_{i, t}$ is the cost of disbanding a unit of type $U_{i}$ at step $t$.

Certainly, for every type $U_{i}, i=1,2, \ldots, q$,

$$
u_{i, t}=u_{i, t-1}+\bar{u}_{i, t}-\tilde{u}_{i, t}, \quad t=1,2, \ldots, T .
$$

For simplicity, the costs $\bar{p}_{i, t}$ and $\tilde{p}_{i, t}$ are calculated for the current step, although in reality payments eventually are made for longer periods of time.

Denote by $\alpha_{j, t}$, for $j=1,2, \ldots, l$, the acceptable level of the negative consequences resulting from scenario $S_{j}$ if it happens during the period $[(t-1) L, t L]$ for $t=1,2, \ldots, T$.

A discrete dynamic model follows: 


\section{Model 5}

$$
\sum_{\mathrm{t}=1}^{\mathrm{T}} \sum_{\mathrm{i}=1}^{\mathrm{q}}\left(p_{i, t} u_{i, t}+\bar{p}_{i, t} \bar{u}_{i, t}+\tilde{p}_{i, t} \tilde{u}_{i, t}\right) \rightarrow \operatorname{minimize}
$$

subject to the constraints

$$
\begin{aligned}
& N_{j}\left(F_{1}\left(u_{1, t}, u_{2, t}, \ldots, u_{q, t}\right), \ldots, F_{n}\left(u_{1, t}, u_{2, t}, \ldots, u_{q, t}\right) \leq \alpha_{j, t}, j=1,2, \ldots, l, t=1,2, \ldots, T,\right. \\
& u_{i, t}=u_{i, t-1}+\bar{u}_{i, t}-\tilde{u}_{i, t}, i=1,2, \ldots, q, t=1,2, \ldots, T \\
& \lambda_{i, t}^{(1)} \leq u_{i, t} \leq \lambda_{i, t}^{(2)}, i=1,2, \ldots, q, t=1,2, \ldots, T \\
& \mu_{i, t}^{(1)} \leq \bar{u}_{i, t} \leq \mu_{i, t}^{(2)}, i=1,2, \ldots, q, t=1,2, \ldots, T \\
& v_{i, t}^{(1)} \leq \tilde{u}_{i, t} \leq v_{i, t}^{(2)}, i=1,2, \ldots, q, t=1,2, \ldots, T \\
& \tilde{u}_{i, t} \leq u_{i, t-1}, i=1,2, \ldots, q, t=1,2, \ldots, T \\
& u_{i, t}, \bar{u}_{i, t}, \tilde{u}_{i, t}-\text { integers, } i=1,2, \ldots, q, t=1,2, \ldots, T \\
& F_{i}\left(u_{1, T}, u_{2, T}, \ldots, u_{q, T}\right) \geq \bar{c}_{i}, i=1,2, \ldots, n .
\end{aligned}
$$

The numbers $\lambda_{i, t}^{(k)}, \mu_{i, t}^{(k)}, v_{i, t}^{(k)}$, for $k=1,2, \quad i=1,2, \ldots, q, t=1,2, \ldots, T$, are nonnegative integers and they define the specific goals, ambitions, or restrictions (if there are any) at any step $t$ for any unit type $U_{i}$. The presence of any of these numbers is optional but they can be put also for the purpose of analysis and/or to aid the computational algorithms. Certainly, whenever $\lambda_{i, t}^{(1)}=\lambda_{i, t}^{(2)}$, or respectively, $\mu_{i, t}^{(1)}=\mu_{i, t}^{(2)}$, $v_{i, t}^{(1)}=v_{i, t}^{(2)}$, for some $i=1,2, \ldots, q, t=1,2, \ldots, T$, we arrive at a constraint from equality type.

Remark: It is presumed that the bounds $\lambda_{i, t}^{(k)}, \mu_{i, t}^{(k)}$, and $v_{i, t}^{(k)}$, for $k=1,2$, $i=1,2, \ldots, q, t=1,2, \ldots, T$, are chosen so that the set defined by the constraints in Model 5 is nonempty. This remark pertains also to all models that follow.

Denote now by $P(t)$ the cost incurred at step $t$ for $t=1,2, \ldots, T$, i.e. the objective function (4) from Model 5.

Let $b_{t}$ be the available budget at step $t$ for $t=1,2, \ldots, T$. The following is a dynamic minimization of the integral negative consequences under budget limitations. 


\section{Model 6}

$$
\max _{1 \leq t \leq T} \sum_{j=1}^{l} w_{j} N_{j}\left(F_{1}\left(u_{1, t}, u_{2, t}, \ldots, u_{q, t}\right), \ldots, F_{n}\left(u_{1, t}, u_{2, t}, \ldots, u_{q, t}\right)\right) \rightarrow \text { minimize }
$$

subject to the constraints

$$
\begin{aligned}
& P(t) \leq b_{t}, t=1,2, \ldots, T, \\
& u_{i, t}=u_{i, t-1}+\bar{u}_{i, t}-\tilde{u}_{i, t}, i=1,2, \ldots, q, t=1,2, \ldots, T, \\
& \lambda_{i, t}^{(1)} \leq u_{i, t} \leq \lambda_{i, t}^{(2)}, i=1,2, \ldots, q, t=1,2, \ldots, T, \\
& \mu_{i, t}^{(1)} \leq \bar{u}_{i, t} \leq \mu_{i, t}^{(2)}, i=1,2, \ldots, q, t=1,2, \ldots, T, \\
& v_{i, t}^{(1)} \leq \tilde{u}_{i, t} \leq v_{i, t}^{(2)}, i=1,2, \ldots, q, t=1,2, \ldots, T, \\
& \tilde{u}_{i, t} \leq u_{i, t-1}, i=1,2, \ldots, q, t=1,2, \ldots, T, \\
& u_{i, t}, \bar{u}_{i, t}, \tilde{u}_{i, t}-\text { integers, } i=1,2, \ldots, q, t=1,2, \ldots, T, \\
& F_{i}\left(u_{1, T}, u_{2, T}, \ldots, u_{q, T}\right) \geq \bar{c}_{i}, i=1,2, \ldots, n .
\end{aligned}
$$

In the above dynamic models, it is allowed at any step units of one and the same type to be disbanded and acquired. If we prohibit such a possibility and also ignore the one-step constraints for the acquired and the disbanded units, then both previous models can be simplified by excluding the variables $\bar{u}_{i, t}$ and $\tilde{u}_{i, t}$ from them. In this case the function $P(t)$ from (4) in Model 5, and respectively, from the set of constraints in Model 6, is replaced by the following one

$$
\hat{P}(t)=\sum_{t=1}^{T}\left(\sum_{i=1}^{q} p_{i, t} u_{i, t}+\sum_{i=1}^{q} \max \left\{0, u_{i, t}-u_{i, t-1}\right\} \bar{p}_{i, t}+\sum_{i=1}^{q} \max \left\{0, u_{i, t}-u_{i, t-1}\right\}\left(\tilde{p}_{i, t}-p_{i, t}\right)\right)
$$

Models 5 and 6 can be modified in order to take into account changes in the force structure after various upgrades of units. Formally, after an upgrade, an old unit is disbanded at a zero cost and the same unit appears as a new unit of a different type acquired at a different cost. We illustrate this in the following models.

Introduce, for $i=1,2, \ldots, q, j=1,2, \ldots, q$ and $t=1,2, \ldots, T$, new variables:

$\delta_{i, j}$ equals 1 if an upgrade from type $U_{i}$ to type $U_{j}$ is possible, or equals 0 if such an upgrade is impossible $\left(\delta_{i, j}=0\right.$ for every $\left.i=1,2, \ldots, q\right)$; 
$v_{i, j, t}$ - the number of units of type $U_{i}$ that are transformed to units of type $U_{j}$ after an upgrade at step $t\left(v_{i, j, t}=0\right.$ for all $t=1,2, \ldots, T$ whenever $\left.\delta_{i, j}=0\right)$;

$g_{i, j, t}$ - the upgrade cost for a unit of type $U_{i}$ to be transformed to a unit of type $U_{j}$ at step $t\left(g_{i, j, t}=0\right.$ for all $t=1,2, \ldots, T$ whenever $\left.\delta_{i, j}=0\right)$.

\section{Model 7}

$\sum_{t=1}^{T} \sum_{i=1}^{q}\left(p_{i, t} u_{i, t}+\bar{p}_{i, t} \bar{u}_{i, t}+\tilde{p}_{i, t} \tilde{u}_{i, t}+\sum_{j=1}^{q}\left(g_{j, i, t}-\bar{p}_{i, t}\right) v_{j, i, t}+\tilde{p}_{i, t} \sum_{j=1}^{q} v_{i, j, t}\right) \rightarrow$ minimize

subject to the constraints

$N_{j}\left(F_{1}\left(u_{1, t}, u_{2, t}, \ldots, u_{q, t}\right), \ldots, F_{n}\left(u_{1, t}, u_{2, t}, \ldots, u_{q, t}\right)\right) \leq \alpha_{j, t}, \quad j=1,2, \ldots, l, \quad t=1,2, \ldots, T$,

$u_{i, t}=u_{i, t-1}+\bar{u}_{i, t}-\tilde{u}_{i, t}, i=1,2, \ldots, q, t=1,2, \ldots, T$,

$\lambda_{i, t}^{(1)} \leq u_{i, t} \leq \lambda_{i, t}^{(2)}, i=1,2, \ldots, q, t=1,2, \ldots, T$,

$\mu_{i, t}^{(1)} \leq \bar{u}_{i, t} \leq \mu_{i, t}^{(2)}, i=1,2, \ldots, q, t=1,2, \ldots, T$,

$v_{i, t}^{(1)} \leq \tilde{u}_{i, t} \leq v_{i, t}^{(2)}, i=1,2, \ldots, q, t=1,2, \ldots, T$,

$\tilde{u}_{i, t} \leq u_{i, t-1}, i=1,2, \ldots, q, t=1,2, \ldots, T$,

$0 \leq \sum_{j=1}^{q} \delta_{j, i} v_{j, i, t} \leq \bar{u}_{i, t}, i=1,2, \ldots, q, t=1,2, \ldots, T$,

$0 \leq \sum_{j=1}^{q} \delta_{i, j} v_{i, j, t} \leq \tilde{u}_{i, t}, i=1,2, \ldots, q, t=1,2, \ldots, T$,

$u_{i, t}, \bar{u}_{i, t}, \tilde{u}_{i, t}, 0 \leq v_{i, j, t}-$ integers, $i, j=1,2, \ldots, q, t=1,2, \ldots, T$,

$F_{i}\left(u_{1, T}, u_{2, T}, \ldots, u_{q, T}\right) \geq \bar{c}_{i}, i=1,2, \ldots, n$.

Denote by $Q(t)$ the objective function of Model 7. The following model for minimization of the integral negative consequences under budget limitations includes upgrades of units.

\section{Model 8}

$\max _{1 \leq t \leq T} \sum_{j=1}^{l} w_{j} N_{j}\left(F_{1}\left(u_{1, t}, u_{2, t}, \ldots, u_{q, t}\right), \ldots, F_{n}\left(u_{1, t}, u_{2, t}, \ldots, u_{q, t}\right)\right) \rightarrow$ minimize 
subject to the constraints

$$
\begin{aligned}
& Q(t) \leq b_{t}, t=1,2, \ldots, T, \\
& u_{i, t}=u_{i, t-1}+\bar{u}_{i, t}-\tilde{u}_{i, t}, i=1,2, \ldots, q, t=1,2, \ldots, T, \\
& \lambda_{i, t}^{(1)} \leq u_{i, t} \leq \lambda_{i, t}^{(2)}, i=1,2, \ldots, q, t=1,2, \ldots, T, \\
& \mu_{i, t}^{(1)} \leq \bar{u}_{i, t} \leq \mu_{i, t}^{(2)}, i=1,2, \ldots, q, t=1,2, \ldots, T, \\
& v_{i, t}^{(1)} \leq \tilde{u}_{i, t} \leq v_{i, t}^{(2)}, i=1,2, \ldots, q, t=1,2, \ldots, T, \\
& \tilde{u}_{i, t} \leq u_{i, t-1}, i=1,2, \ldots, q, t=1,2, \ldots, T, \\
& 0 \leq \sum_{j=1}^{q} \delta_{j, i} v_{j, i, t} \leq \bar{u}_{i, t}, i=1,2, \ldots, q, t=1,2, \ldots, T, \\
& 0 \leq \sum_{j=1}^{q} \delta_{i, j} v_{i, j, t} \leq \tilde{u}_{i, t}, i=1,2, \ldots, q, t=1,2, \ldots, T, \\
& u_{i, t}, \bar{u}_{i, t}, \tilde{u}_{i, t} 0 \leq v_{i, j, t}-\text { integers, } i, j=1,2, \ldots, q, t=1,2, \ldots, T, \\
& F_{i}\left(u_{1, T}, u_{2, T}, \ldots, u_{q, T}\right) \geq \bar{c}_{i}, i=1,2, \ldots, n .
\end{aligned}
$$

Solving numerically the above stated models can be a significant challenge. This is mainly due to the integer requirement imposed on the decision variables, but the scaling (especially in the dynamic setting) can bring more difficulties. Discussing numerical techniques for these problems falls beyond the scope of the present paper.

\section{Conclusion}

The head on formulation and in particular the solution of the optimization problem may pose insurmountable challenges, not only due to scaling and required computational resources. Among the difficulties might be lacking standardization of tasks, units, and capability descriptions, our ability to estimate future costs of acquiring, maintaining, and disbanding units, the amount of effort required to estimate negative effects over the sets of planning scenarios and force structures, in particular for the dynamic models, etc.

Therefore, we currently consider application of this approach to support planning in a more limited scope, e.g. in designing a particular subset of the force structure. Among other advantages, this approach would allow for better understanding of the sensitivity of the solution to variety of uncertainties. 
More importantly, this approach contributes to the insight of planners and decision makers. It contributes to the elaboration and enhances the understanding of force planning problems, information needs, and decision-making requirements. Finally, with adequate visualization and information systems support, the application of the approach will promote rational decision-making towards effective and efficient transformation of the armed forces of Bulgaria.

\section{Acknowledgement}

The article presents interim research results from the study SfP 981149 "Operations Research Support to Force and Operations Planning in the New Security Environment," partially financed by NATO within its Science for Peace program.

\section{Notes:}

1 Handbook on Long Term Defence Planning, RTO Technical Report 69 (Paris: NATO Research and Technology Organization, April 2003), <www.rta.nato.int/Pubs/RDP.asp? RDP=RTO-TR-069> (25 November 2007).

2 Henry Bartlett, G. Paul Holman, and Timothy E. Somes, "The Art of Strategy and Force Planning," in Strategy and Force Planning, $4^{\text {th }}$ ed. (Newport, R.I.: Naval War College Press, 2004), 17-33.

3 Todor Tagarev, "Methodology for Defense and Force Planning," in Methodology and Scenarios for Defence Planning (Sofia: Military Publishing House, 2007), 179-207.

4 Or "planning situation" in NATO terms.

5 Such capability partition did not exist prior to the UMSSOFT 2007 conference. An expert proposal was developed soon afterwards and currently waits for an official sanction. Details on the proposed partition are provided in Todor Tagarev and Valeri Ratchev, Bulgarian Defense Policy and Force Development 2018 (Sofia, Military Publishing House, 2008). in Bulgarian.

6 The first library of this type in Bulgaria was created at the end of 2007. See Tagarev and Ratchev, Bulgarian Defense Policy and Force Development 2018.

7 Or "future challenges risks" in U.S. terminology. See The National Defense Strategy of the United States of America (Washington, D.C.: Department of Defense, March 2005), p. 11, <www.defenselink.mil/news/Mar2005/d20050318nds1.pdf> (25 November 2007). 
8 For details the reader may refer to Guide to Capability-Based Planning, TR-JSA-TP3-22004 (The Technical Cooperation Program, Joint Systems and Analysis Group, Technical Panel 3, MORS Workshop, October 2004), <www.mors.org/meetings/cbp/read/TP3_CBP.pdf> (25 November 2007).

9 See Guide to Capability-Based Planning; Ian Wilson and Bill Ralston, Scenario Planning Handbook: Developing Strategies in Uncertain Times (Mason, OH: Thomson SouthWestern, 2006); Paul K. Davis, Steven C. Bankes, and Michael Egner, Enhancing Strategic Planning with Massive Scenario Generation: Theory and Experiments, TR-392 (Santa Monica, CA: RAND National Security Research Division, 2007), <www.rand.org/pubs/ technical_reports/TR392> (25 November 2007); Methodology and Scenarios for Defence Planning.

TODOR TAGAREV is Head of the Defence and Force Management Department of "G.S. Rakovski" Defence and Staff College in Sofia Bulgaria and member of NATO's Research and Technology Board. He was the first Director of the Defence Planning Directorate since its establishment in early 1999. From May until late 2001, he served as Director for Armaments Policy in the Bulgarian Ministry of Defence and National Armaments Director. Among other duties, he coordinated all defence modernization and R\&D programs in support of defence reform and NATO integration. He graduated from the Bulgarian Air Force Academy in 1982 and received a PhD degree in systems and control from Zhukovsky Air Force Engineering Academy, Moscow, in 1989. Dr. Tagarev is a 1994 Distinguished Graduate of the US Air Command and Staff College at Maxwell Air Force Base, Ala. E-mail: tagarev@gmail.com.

TSVETOMIR TSACHEV is Senior Research Fellow in the "Operations Research" Department of the Institute of Mathematics and Informatics at the Bulgarian Academy of Sciences. In 1983 he graduated the Faculty of Mathematics and Mechanics (now Faculty of Mathematics and Informatics) of the Sofia University. In 1987 he finalized his doctoral studies in the Institute of Mathematics and received a PhD degree in mathematics. Dr. Tsachev was a visiting professor to the University of Delaware in the U.S. and, more recently, to Austrian and German research organizations. His main fields of interest are optimal control and operations research, with additional interests in variational methods in nonlinear analysis and mathematical economics. Address: Acad. G. Bonchev str., block 8, 1113 Sofia, Bulgaria. Email: tsachev@math.bas.bg.

Dr. Tagarev and Dr. Tsachev are among the co-directors of SfP 981149.

NIKOLAY ZHIVKOV is Senior Researcher in the "Operations Research" Department of the Institute of Mathematics and Informatics at the Bulgarian Academy of Sciences. His recent work involves application of operations research methods and techniques to scenario-based, capabilities-oriented planning and to forecasting demands and managing supply chains during crises.E-mail:niz@abv.bg. 